Revista Mídia e Cotidiano

Artigo Seção Livre

Volume 11, Número 3, dezembro de 2017

Submetido em: 27/07/2017

Aprovado em: 06/11/2017

\title{
GAMERGATE: cultura dos jogos digitais e a identidade gamer masculina
}

\section{GAMERGATE: digital games culture and male gamer identity}

\author{
Lucas GOULART ${ }^{1}$; Henrique NARDI ${ }^{2}$
}

\section{Resumo:}

Tomando como ponto de partida o Gamergate - evento da cultura de jogos digitais onde mulheres, LGBTQs e pessoas não-brancas foram hostilizadas e agredidas por sua atuação dentro da cultura de jogos digitais - dissertamos a respeito de como essas culturas se relacionam com a identidade do "homem nerd". Compreendemos que esse evento é uma reação à possível mudança dessas culturas rumo a uma possibilidade mais inclusiva, o que colocaria em cheque tais identidades. Dessa maneira, investigamos como historicamente essa cultura se centra na promoção e "venda" dessa identidade gamer masculina, e como movimentos inclusivos e centrados em diversidade sexual e de gênero possibilitam diferentes impactos e acessos a esses contextos.

Palavras-chave: jogos digitais, masculinidades, gênero, sexualidade.

\begin{abstract}
:
Taking the "gamergate controversy" as a starting point, we analyze how digital gaming cultures relates itself to male "nerd / geek" identity. We understand this event as a reaction to the constant changing of the culture towards a more inclusive stance - which is perceived as a menace to this identity formation. Being so, we investigate some of the history of gaming culture, how it promotes the male nerd identity, and how inclusive communities and gaming movements focused on sexual and gender diversity impacts these contexts.
\end{abstract}

Keywords: digital gaming, masculinities, gender, sexuality.

\footnotetext{
${ }^{1}$ Possui doutorado em Psicologia Social e Institucional pela Universidade Federal do Rio Grande do Sul (2017). Tem interesse de pesquisa nos seguintes temas: gênero, sexualidade, teoria queer, jogos digitais e novas tecnologias de informação. E-mail: la_goulart@hotmail.com.

${ }^{2}$ Doutor em Sociologia pela Universidade Federal do Rio Grande do Sul (2002), Professor Associado nessa mesma instituição e atualmente diretor do Instituto de Psicologia. É coordenador do Núcleo de Pesquisa em Sexualidade e Relações de Gênero (NUPSEX) e do Centro de Referência em Direitos Humanos (CRDH). E-mail: hcnardi@gmail.com.
} 
Introdução

Em 2013, o autor e designer de jogos digitais Eric Zimmerman publicou o que ele chamou de "Manifesto Por Um Século Lúdico" (ZIMMERMAN; CHAPLIN, 2013), onde advoga por uma nova maneira de enxergar o que seriam os jogos digitais. Zimmerman reconhece que o século XX viu a ascensão de duas principais tendências: a centralidade das teorias de produção, acesso, reconhecimento e distribuição de informações, e, a primazia da imagem em movimento como forma cultural dominante. Contudo, para o autor, o século XXI seria o século dos jogos - principalmente dos jogos digitais. Embora os jogos em geral tenham uma importância organizatória para as relações sociais dentro da história da humanidade, nunca a necessidade de compor o lúdico como linguagem teve tanto valor quanto no século XXI. Para o autor, esse "lúdico" estabeleceria uma nova possibilidade de visualização das dinâmicas contemporâneas de processamento da informação, onde mais do que simplesmente compreender "passivamente" esses sistemas, é necessário pensar como designers, levar em consideração as arquiteturas ali presentes, sua não-linearidade e os efeitos e impactos que diferentes arranjos compõem aos sistemas nos quais estão inseridos. Em consonância a isso, os jogos digitais acabaram se tornando uma indústria bilionária. Apenas no ano de 2016, de acordo com o relatório do site SuperData Research (2017), o mercado de jogos digitais faturou cerca de US\$ 91 bilhões.

Sendo uma indústria tão prolífica e promissora, os jogos digitais poderiam ser considerados - em um primeiro momento, ou para um observador casual - como o melhor exemplo dos ciclos de produção e consumo do capitalismo tardio: uma intersecção entre os ciclos de inovação tecnológica, consumo e descarte constante, onde a "novidade" parece ser o centro de todo o projeto de experiência (AMARAL; DE PAULA, 2007). Dentro desse pensamento, é de se imaginar que a máxima das mídias de massa - "temos algo para todo mundo" - poderia ser considerada a "centralidade ética" dessa cultura. Afinal de contas, a própria difusão dos jogos em diferentes espaços, mantidos por diferentes redes de sociabilidade e sustentação monetária, e compreendendo diferentes simulações aparentemente seguiria a tendência - como o cinema, televisão e literatura de públicos-alvo diferentes, ocupando espaços distintos e compondo uma cultura plural. 
Contudo, é impossível compreender os jogos digitais sem compreendermos sua cultura e história - e o jogo de forças e diferentes interesses e influências que essas exercem, criando possibilidades e impossibilidades de acesso, expressão e convivência dentro das culturas de jogos digitais. Nunca tal realidade ficou tão evidente quanto no evento que ficou conhecido como Gamergate - um ataque coletivo organizado contra mulheres e outras personalidades que advogavam por uma cultura de jogos digitais mais inclusiva e diversa. Dessa maneira, esse artigo tem como objetivo analisar as relações entre as culturas de jogo digital enquanto contexto tóxico, o Gamergate enquanto evento e a produção de identidades masculinas gamers - assim como as cenas de resistência que tem surgido em resposta a esses eventos.

\section{A Cultura de Jogos Digitais Como Tecnocultura Tóxica}

Embora de difícil definição a priori (como qualquer cultura midiática ligada ao mundo digital na chamada web 2.0) e sendo altamente mutante e heterogênea, presume-se que a análise das culturas de jogos digitais deva levar em consideração que os jogos, assim como as mídias que os antecederam (filmes, séries, literatura, quadrinhos), sua organização por gêneros e subgêneros, ambientes, e diferentes possibilidades de acesso estabelecidos historicamente. Essa história é pautada por diversos marcadores: como esses gêneros são (e já foram) jogados - e narrados -, quais são os objetivos desses jogos, que tipo de histórias esses contam, quais suas ligações com gêneros literários específicos, quais suas ligações com outros jogos específicos, como são seus gráficos, quais são as empresas que os produzem (e por quais tipos de jogo/franquias essas são reconhecidas), que tipo de experiência esses oferecem, em quais plataformas estão disponíveis, etc. Assim, seria impossível pensar em um jogo digital que seja constituído sem ter como referência essa organização pregressa (de regras, de histórias, de gráficos) que já orientaria essas experiências de alguma maneira, compondo assim uma instância coletiva. Nessas instâncias coletivas se produzem também conteúdos próprios, como piadas, histórias (relatos pessoais, narrativas nos universos dos jogos, etc.), ilustrações, quadrinhos, vídeos (desde animações vídeos que mostram a performance de 
jogadores/as) sobre as performances, experiências, narrativas e mecânicas do jogar compreendendo isso então como cultura de jogo digital (SQUIRE, 2006).

Contudo, apesar de heterogênea e múltipla, a cultura dos jogos digitais tem o que Michael Warner (2003) define como "público" - ou seja, um "indivíduo padrão" característico, que se materializa com mais facilidade como referência tanto culturalmente como em termos de produção, indústria e público-alvo. O "público" dos jogos digitais seriam, então, os homens cisgêneros, heterossexuais e brancos. Nas duas primeiras gerações dos jogos digitais - do final da década de 1970 até o meio da década de 1980 - os jogos digitais tinham públicos alvos mais variados, mas sendo considerado (como era a televisão nessa época) algo mais voltado para "família". Contudo, com o crash do videogame norte-americano de 1983 - crise econômica, onde a indústria quase faliu - começa-se a se privilegiar cada vez mais para homens jovens (DONOVAN, 2010). A ideia do "clássico" jogo digital como violento, sexista, onde o papel das mulheres é simplesmente ser resgatada (como um "prêmio sexual”), e por vezes homo/transfóbico (ou que, pelo menos, invisibiliza sexualidades não-heterossexuais ou identidades de gênero não-cisgêneras) se consolida com os chamados "temas maduros" dos anos 1990. Esses eram jogos graficamente mais violentos e considerados mais "chamativos" para esses públicos. Tal tendência alcançou o seu ápice nos anos 1990 e 2000 (GRAY; BUYUKOZTURK; HILL, 2017). Dessa época gestou-se a maioria das ideias do que seria o jogo digital contemporâneo - ideia que ainda vigora no mainstream: jogos extremamente violentos, com mecânicas e sistemas voltados à simulação bélica (seja essa calcada em um viés "realista" ou "cinematográfico") onde as personagens femininas deveriam ser, ao mesmo tempo, um chamariz sexual e um prêmio ao jogador (tropo comumente nomeado como "donzela indefesa"). Assim, de acordo com Anna de Miguel e Montserrat Boix:

Na verdade, estes jogos remetem à socialização no uso da violência como um dos traços-valores mais estreitamente associados à virilidade - violência ilegítima no caso dos "maus" e legítima no caso dos "bons". De alguma forma os computadores começam a ser vistos desde a infância como um segregado componente de gênero, começam a converter-se em "toys for the boys". (DE MIGUEL; BOIX, 2013, p. 51). 
Essa composição de público também tornaria esses espaços no que Adrienne Massanari (2015) chama de tecnoculturas tóxicas. Para a autora, essas tecnoculturas são locais onde a manutenção da centralidade de identidades mantém um patamar de privilégios, com melhor acesso a recursos, criação de comunidades e proteção, enquanto outros sujeitos, principalmente aqueles que mais "se distanciam" dessa compreensão (mulheres, pessoas LGBTQ, pessoas não-brancas) acabam encontrando menos potência e mais vulnerabilidade à violência nesses espaços. Donna Haraway (2000) nomeia essa dinâmica como "informática da dominação". Esse "poder" não seria simplesmente composto por questões de ordem simbólica e social, mas também técnica. Grande parte desses espaços é pensada como "locais livres" para a proteção de diversas identidades sociais, ainda encarnados na crença tecnoutópica da descorporificação total da internet. A manutenção dessa crença é calcada em uma compreensão liberal humanista, onde as diferentes corporificações de gênero, sexo e raça - e os distintos espaços que os corpos ocupam nos espaços sociais - podem ser ignoradas com um discurso de pretensa igualdade que, na realidade, ignora a diferença (HAYLES, 1999).

Assim sendo, questões de dificuldade de acesso, manutenção de privilégios masculinos, misoginia e pouca representatividade feminina e LGBTQ são historicamente pautadas dentro das culturas de jogo digital. Ainda assim, embora estudos, movimentos e produções questionando o lugar das mulheres e meninas dentro dessas culturas tenham mais de 20 anos (CASSEL E ; JENKINS, 1998; KAFAI; HEETER; DENNER; SUN, 2008; KAFAI; RICHARD; TYNES, 2016), essas compreensões sempre se mantiveram ligadas a questões “externas” à cultua de jogos digitais. Por exemplo, relações entre essas culturas e o gender gap tecnológico (IMHOF; VOLLMEYER; BEIERLEN, 2007), mulheres e tecnologia (PLANT, 1999), ou a iminência do chamado cyberbullying misógino e homo / transfóbico (CONDIS, 2014) sempre foram prevalentes e visíveis. Contudo, a novidade do chamado Gamergate foi visibilizar, de forma efetiva, que existe uma composição opressiva singular a essas culturas, de jogo um ambiente não planejado e que encontra dificuldades para compor espaços diversos, assim como a existência de grupos ativos, treinados, e bem colocados nessa cultura dispostos a manter as relações de dominação masculina, cisgênero e heterossexual nessa cultura. 


\section{O evento Gamergate}

O chamado Gamergate se inicia em agosto de 2014 quando o game designer Eron Gjioni escreve um longo texto sobre o final de seu relacionamento com um/a também game designer chamado/a Zoe Quinn. De acordo com Gjioni, Quinn estaria se relacionando sexualmente com críticos de jogos digitais para que conseguisse boas críticas de seu jogo (gratuito) a respeito de sua vivência com depressão, chamado Depression Quest. Após esse fato, Quinn começou a receber ameaças de morte e estupro, tendo seus dados revelados na internet (uma prática conhecida como doxxing) e contas em redes sociais invadidas. Dessa maneira, compôs-se um movimento que tinha como objetivo (pretensamente) zelar pela "ética no jornalismo dos jogos digitais", visto como corrupto e seriamente prejudicado pela proximidade entre produtores e críticos/as. Tal evento foi nomeado dessa forma visto que o sufixo gate (relacionado ao caso Watergate ${ }^{3}$ ) remete a uma conspiração, nomenclatura que acabou sendo usada para identificar esse grupo que, pretensamente, lutaria contra uma conspiração entre produtores e game designers independentes (principalmente aqueles ligados à divulgação da diversidade sexual, de gênero e de raça na cultura de jogos digitais) (CHESS; SHAW, 2015; MASSANARI, 2015; SALTER, 2017). Na prática, o Gamergate investiu muito mais no ataque principalmente misógino, mas também homofóbico, transfóbico e racista a trabalhadoras/es da indústria, crítica e demais membros da cultura de jogos digitais (BURGESS; METAMORO-FERNANDÉZ, 2016). Suas principais vítimas, além de Zoë Quinn, foram a crítica feminista de jogos Anita Sarkeesian e a game designer Brianna $\mathrm{Wu}$. Enquanto ambas sofreram as mesmas ameaças e vazamento de informações de Quinn, Sarkeesian teve cancelar uma palestra que daria na Utah State University por essa sofrer uma ameaça de tiroteio em massa (AHMED; MARCO, 2014), enquanto Wu teve de fugir de sua casa após receber ameaças de estupro citando seu endereço residencial algo que também aconteceu com seus parentes próximos (TOTLO, 2014).

\footnotetext{
${ }^{3}$ Watergate foi um escândalo político ocorrido em 1972 nos Estados Unidos, onde documentos foram roubados de um prédio pertencente ao Partido Democrata. Uma longa conspiração que ligava tais crimes à administração presidencial foi revelada, sendo então o prefixo "gate" utilizado para sinalizar conspirações dentro da cultura estadunidense.
} 


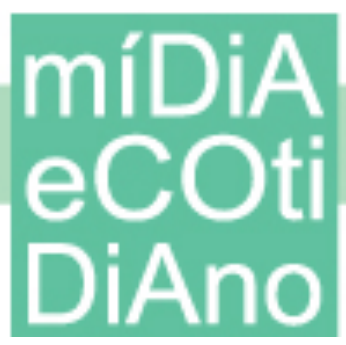

\section{PPGMC}

uff
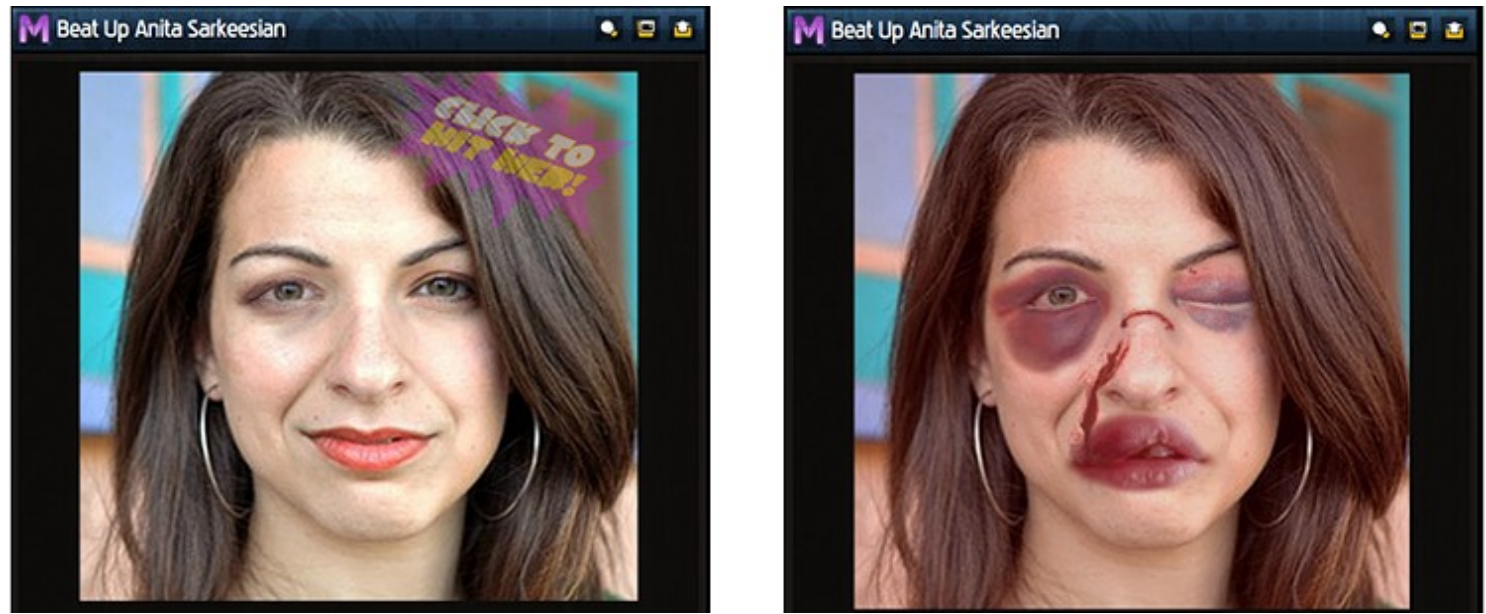

Jogo produzido em flash onde é possível agredir a crítica Anita Sarkeesian (ROUNER, 2014). Disponível em http://www.houstonpress.com/arts/8-criticisms-of-anita-sarkeesian-that-are-utter-bullshit-6382966

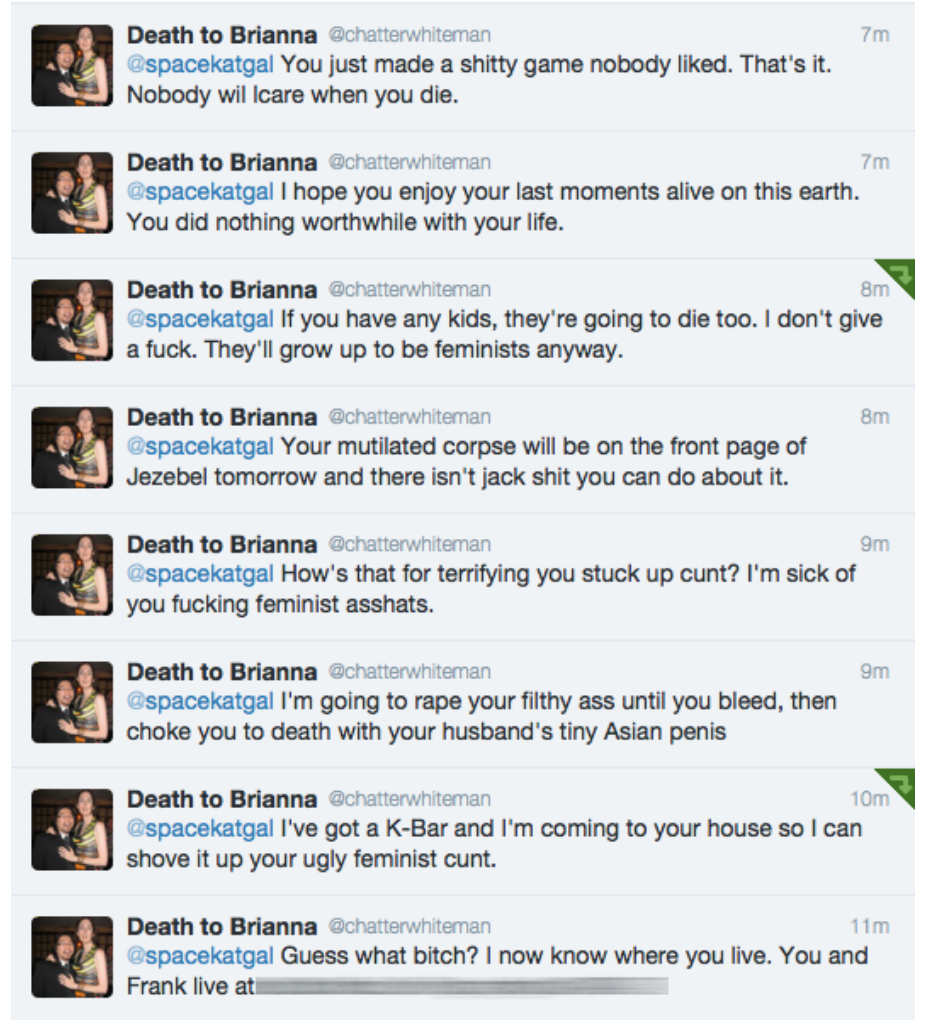

Ameaças de morte contra a desenvolvedora Brianna Wu (GAMERANX, 2014), disponível em http://gameranx.com/updates/id/24642/article/game-developer-brianna-wu-driven-from-home-afterdeath-threats-and-doxxing/) 
Embora seja um evento iniciado nos Estados Unidos, tal contexto também tomou proporções nas culturas de jogo digital brasileiras. Como efeito da exposição causada pelo Gamergate, algumas mulheres teceram críticas ao modo como as comunidades de jogos digitais eram hostis e tóxicas a presença delas, além de críticas mais diretas a certos membros eminentes da comunidade de jogos digitais por suas posições machistas e homofóbicas (PARTIDO PIRATA, 2015). Essas foram ameaçadas, assediadas e tiveram seus dados expostos em fóruns públicos (PORTAL IMPRENSA, 2015). Tais histórias foram reveladas também em um documentário curta metragem universitário chamado "Player 2", que foi disponibilizado no Youtube em julho de 2015. Contudo, as realizadoras do documentário também sofreram o mesmo tipo de assédio e vazamento de informações das mulheres às quais retrataram após o lançamento do mesmo, escolhendo então por retirar o filme da plataforma como medida de proteção (GERAÇÃO GAMER, 2015).

O Gamergate acabou por explorar essas ferramentas para visibilizar as suas discussões, localizar seus alvos e trocar informações sem perigo de serem reconhecidos e responsabilizados por isso. Utilizando-se de uma compreensão do algoritmo dos sites de discussões anônimo Reddit e $4 \mathrm{chan}^{4}$ - onde esses sabiam como organizar-se para deixar as discussões e seus pontos de vista sempre visíveis, enquanto dificultavam respostas contrárias utilizando-se falhas e detalhes do funcionamento desses sites - os partidários do Gamergate puderam, em um primeiro momento, fazerem-se ouvidos e calar aqueles contrários a ele. Ao mesmo tempo, tentativas de visibilizar opiniões contrárias em relação ao Gamergate em plataformas com o Twitter - utilizando-se da hashtag \#gamergate ${ }^{5}$ - acabou por visibilizar indivíduos, e apontar novos alvos para assédio, ameaças e doxxing por parte do movimento. A tentativa de criar hashtags

\footnotetext{
${ }^{4}$ Reddit e 4chan são fóruns anônimos da internet, que são - na grande maioria das vezes - pouco reguladas. As particularidades dessas culturas e como essas influenciam as culturas de jogo digital serão discutidas no capítulo 5.
} 
contrárias - como \#stopgamergate2014, criado pelo jornalista Verendeer Jubal ${ }^{6}$ - também eram monitoradas e prontamente atacadas.

Contudo, o que faz com que o Gamergate ascenda nesse exato momento são importantes debates, mudanças e retomadas dessa história diversa nas culturas de jogo digital. A misoginia e a repetição dos temas tipicamente masculinos nos jogos (tanto representativamente quanto suas mecânicas) são pauta de movimentos desde o início dos anos 1990, quando foi gestado o chamado "Girl Games Movement" - um movimento conjunto entre jogadoras/es, game designers, ativistas feministas e acadêmicas/os, que tinham como objetivo resistir aos jogos que o mercado produzia para meninas (os chamados pink games, jogos que simulavam tarefas pretensamente "femininas", como jogos de moda ou de afazeres domésticos) (CASSEL; JENKINS, 1998, GOULART, 2014). Embora tal movimento tenha acabado no início dos anos 2000 - quando os estúdios de produção de jogos ligados a esse movimento acabaram por falir (antes do advento dos jogos indie) - sua influência, se não sobre a indústria, mas sobre outras esferas da cultura de jogos digitais, perdurou - em um primeiro momento academicamente, mas influenciado outras esferas posteriormente (KAFAI; HEETER; DENNER; SUN, 2008).

Essa zona de influência acabou alargando-se e atingindo diretamente a crítica e jornalismo especializados em jogos, que acabaram por passar por uma mudança de paradigma (PERREAULT; VOS, 2016). Essa havia sido inicialmente planejada e articulada como uma veiculação de esclarecimento "objetivo" em relação aos produtos e, mais tarde, como um guia de estratégias e dicas para uma performance mais potente, quando essa ainda era organizada por mídia impressa (MENDES, 2006). Contudo, desde a difusão da web 2.0, a crítica em jogos digitais - influenciada por discussões não apenas relativas ao gênero (e à violência), mas também ao surgimento dos Game Studies acadêmicos no início dos anos 2000 - acabou se preocupado com leituras sociais dos jogos e mecânicas, e de circuitos de produção alternativos. Com o tempo, a crítica de

\footnotetext{
${ }^{6}$ Jubal, um homem sikh, acabou sendo vítima de montagens e boatos que o ligavam aos atentados de Paris em novembro de 2015. Tais boatos foram comprovadamente ligados às plataformas, locais e táticas dos defensores do Gamergate. (LEE, 2015).
} 
jogos digitais passou de um "guia do consumidor" para um jornalismo interessado em outras possibilidades e experiências possíveis aos jogos digitais, focado principalmente na cena indie, visto que essas cenas seriam aquelas mais interessadas em organizar diferentes usos dos jogos digitais (JAGODA, 2017). Dessa forma, boa parte dos/as jogadores/as resistentes a essas mudanças começaram a se utilizar de outras plataformas, principalmente os chans (reddit, 4chan, 8chan, etc.) e o Youtube. Ao mesmo tempo, a indústria tem reconhecido a necessidade de se voltar para o "público LGBTQ", inserindo possibilidades de relacionamento entre pessoas do mesmo sexo em variados jogos embora elas sejam sempre "alternativas" a uma narrativa linear tipicamente heterossexual (CONDIS 2014; SHAW 2014).

Essas mudanças de paradigma e perspectiva das produtoras não agradaram aos/às jogadores/as mais tradicionais, e não coincidentemente são razões claras da necessidade do Gamergate, de acordo com jogadores/as ligados/as ao movimento. Para esses, a mudança da crítica em relação à mídia é uma prova de que existiriam grupos organizados com apenas um objetivo: destruir os jogos digitais. A organização de comunidades por meio de teorias da conspiração - como é de praxe da direita estadunidense - são importantes formas de comunicação entre os membros do Gamergate (CHESS; SHAW, 2015), onde esses se veem, ao mesmo tempo, como os únicos que conseguem ver a verdade, como os heróis que mantém um "estilo de vida" e de que são as verdadeiras vítimas de uma guerra cultural, que não seria contra uma indústria e uma organização excludente e opressora, mas contra a própria identidade desses/as jogadores/as. Por trás dessa conspiração, estariam os chamados SJW - "Social Justice Warriors" 7 - pessoas ligadas a movimentos sociais e pela diversidade, lidos como doutrinadores/as e autocráticos/as. Dessa maneira, movimentos como o Gamergate enxergam-se como pessoas lutando pela liberdade, reconhecendo como inimigos aqueles que querem obriga-los a levar em consideração e refletir a respeito de relações de opressão e privilégio (NAGLE, 2017). Não por coincidência, as táticas, estratégias e ideologias de tais movimentos acabam por se repetir na chama alt-right norte americana, reconhecidamente ligada à movimentos extremistas anti-feministas e de supremacia

\footnotetext{
${ }^{7}$ Pode ser traduzido como "Guerreiros/as da Justiça Social"
} 
branca, considerados cruciais para a eleição de Donald Trump à presidência estadunidense (SALTER, 2017).

\section{Entre Consumidores e Nerds - A Identidade Gamer Masculina}

Assim, embora seja um grupo específico, o que é interessante a respeito dos membros do Gamergate é que esses demonstram o quanto a utilização e exploração de certas facetas do sistema de informação dentro da cibercultura ainda privilegia posicionamentos feitos por homens, brancos, cisgêneros e heterossexuais contra as possibilidades de questionar sua hegemonia. Assim, embora os "membros" do Gamergate não sejam tão numerosos - estimativas, ainda que muito vagas, estimam entre 38 e 110 mil membros (MORTENSEN, 2016), é compreensível como esse grupo - por mais que possa ser considerado "minoritário" - tenha sua voz ouvida de forma tão veemente pelas empresas de tecnologia. Empresas como a Intel e Adobe retiraram seus anúncios de sites ligados a críticas ao Gamergate como Kotaku, Gamasutra e Polygon. Isso porque historicamente por ser o "público" da indústria de jogos mainstream, o "gamer" (homem, cisgênero, heterossexual), sua identidade é composta principalmente como um consumidor de jogos. Essa faceta "consumidora" é central para a identidade gamer - a ponto de críticos como Mathew Seiji Burns (2014) utilizarem a expressão "consumidorrei" para definir essa relação - onde os jogadores que se consideram mais "tradicionais" (ou, "os verdadeiros jogadores") sentem que, podendo eles consumir os jogos mais caros (os chamados AAA) esses não conseguem ver diferença entre os produtos constituídos pelas grandes corporações e a existência (ou possibilidade) de uma cultura de jogos digital mais ampla. Essa tendência se mostra ainda mais aguda quando vemos a ascensão de outras plataformas de jogo, como o jogo em mobile e os chamados "jogos casuais" (jogos com uma acessibilidade maior e baixa curva de aprendizado). Isso porque essa identidade acaba se atrelando não apenas ao consumo, mas o quanto se investe nessa monetariamente e em relação ao tempo dispendido (JULL, 2009). Estudos estatísticos recentes demonstram que a ascensão dos jogos mobile inverteu a pirâmide do gênero em relação ao jogo digital, sendo que $52 \%$ do público consumidor de jogos é composto por mulheres, tanto nos Estados Unidos (JAYANTH, 2014) quanto no Brasil (PESQUISA GAME 
BRASIL, 2016). Entretanto, essas pessoas, por não enxergarem-se dentro da demografia estabelecida pela "identidade gamer" ou mesmo por não compreenderem que os jogos mobile sejam jogos "válidos"- por seu investimento restrito - acabam não se não tencionando os fundamentos dessas identidades (SHAW, 2012)

Aa identidades constituídas como "consumidor-rei" acaba por manter uma dificuldade dos/as jogadores/as mais tradicionais de saírem desse papel de consumidores. No momento em que a cultura se comporta de uma maneira diferente do produto que esses “pagaram" (e rompendo com o Boyhood), onde esses são retirados de um espaço de intensa repetição das mesmas temáticas e mecânicas, existiria uma quebra de contrato. Dessa maneira, os produtos da cultura de jogos digitais passam a ser a própria identidade gamer - e a reiteração da experiência masculina hegemônica. Essa relação entre consumo e identidade nos remete à ideia de identidades pret-a-porter de Suely Rolnick. A constituição dessas identidades mediadas pelos bens de consumo do capitalismo tardio seria uma das maneiras de lidar com as perturbações sociais inerentes. A assunção dessas identidades teria então um efeito estabilizador do sujeito, mas tendo como preço a necessidade de resistir às mudanças (ou mesmo a possibilidade de diferença). De acordo com Rolnick:

As forças, ao invés de serem produtivas, ganham um caráter diabólico; o desassossego trazido pela desestabilização torna-se traumático. Para proteger-se da proliferação das forças e impedir que abalem a ilusão identitária, breca-se o processo, anestesiando a vibratilidade do corpo ao mundo e, portanto, seus afetos. (ROLNICK, 1997, p. 20)

Essa identidade reflete, então, em um espaço que Derek Burrill (2008) nomeou como "Boyhood". Para o autor, Boyhood seria, ao mesmo tempo, uma posição de sujeito, uma facilidade de acesso a certas tecnologias e um contexto que favoreceria homens cisgêneros (em sua maioria brancos e heterossexuais) dentro das culturas de jogos digitais. Isso porque a maioria dessas culturas acaba por constituir-se dentro de estruturas de significado que encontram eco em uma socialização masculina (que inclui também certos segmentos de filmes, séries, literatura e quadrinhos). Essa socialização masculina juvenil acaba por reencenar os chamados "mitos de passagem" para que um menino se torne um homem - um ser criado como forte, raivoso, violento, conquistador e dominador 
(de mulheres e de outros homens). Contudo, na era pós-industrial do capitalismo tardio, esses ritos de passagem acabam se restringindo a uma fantasia, um modelo de masculinidade romantizado que se tornou impossível de ser alcançado. Dessa maneira, no contexto das culturas de jogo digital, o Boyhood se torna um valoroso instrumento simbólico para a masculinidade ocidental: essa compõe um local fantasioso onde podese "provar" sua hombridade, reencenando as atividades "clássicas" da fantasia romantizada do homem (guerra, conquista, sacrifício, salvamento de mulheres e crianças) sem ter de lidar com as possíveis falhas desses projetos idealizados (dor, morte, falha ou mesmo alteridade) (BURRILL, 2008).

Essa manutenção da identidade gamer vem de encontro às outras maneiras na qual a cibercultura organiza as masculinidades, principalmente aquelas chamadas como "masculinidades geek (ou nerds)". Lori Kendall (2002; 2011) nos aponta que a identidade de "homem nerd" seria de um homem que não necessariamente alcançaria o ideal "hegemônico" - ou seja, ele se reconhece como fisicamente fraco e socialmente inepto, mas compensaria isso com sua "inteligência" e pretensa expertise na área de tecnologia. A construção dessa imagem de "nerd" provém de narrativas que o identificam assim desde a década de 1960, sendo então composta pela figura do homem branco, heterossexual, cisgênero que preza a inteligência além do físico. A própria linguagem tecnocientífica é constituída por homens para homens, mantendo-a enquanto construto de dominação. Dessa maneira, as possibilidades de acesso de outros sujeitos não-masculinos se torna limitada, e até mesmo escassa. Essa identidade / estereótipo implicaria que:

(1) Computadores são um tipo de tecnologia importante, porém problemática (2) Nerds entendem e gostam de computadores (3) Aqueles que entendem e gostam de computadores são nerds (4) Nerds são socialmente ineptos e sexualmente indesejáveis (5) Nerds são homens brancos. Se tomarmos essas prerrogativas como um todo, elas implicam em duas questões importantes a respeito de raça, gênero e tecnologia. A primeira é que a masculinidade dos nerds ainda está em questão, protegendo uma forma de masculinidade hegemônica que ainda prima pela agressividade e fisicalidade. A segunda é que mulheres e pessoas nãobrancas são excluídas dessa categoria, protegendo um status econômico e tecnológico superior dos homens brancos (KENDALL, 2011, p. 519) 
Por esse motivo a crítica Liz Ryerson (2014) define que a figura central desses movimentos é a do "menino gênio" - um "nerd" da mesma maneira exposto por Lori Kendall, mas que "descobre" e "redescobre" formas de compor tecnologia de maneira que essa nunca se organize de uma maneira crítica ou questionadora do sistema, individualizando completamente o ato de criação tecnológica - afastando a experiências dos jogos das possibilidades de alteridade. Tal compreensão vem de encontro com a ideia de McKenzie Wark (2007) que afirma que a lógica dos jogos se propõe a ser emocionalmente "neutra", compreendendo a ideia de "racional" da masculinidade nerd. Exemplos de como essa lógica de "jogo" enquanto emocionalmente neutra e deslocada de uma ética coletiva é possível de ser visualizada em como é composta pelas "comunidades de sedução" ". Essa sedução é conceituada como a utilização de mecanismos inconscientes para seduzir mulheres, cujo sucesso depende do não investimento emocional, e da centralidade dessa ideia como um sistema de simulação centrado na vitória (ALMOG; KAPLAN, 2015). Não por coincidência, os "SJWs" acabam não sendo apenas culpados por investir em diversidade (representativa e de produção) no meio, mas também por investir em mecânicas e estilos de jogo diferenciais - ou seja, fazendo "jogos ruins", ou ainda "não jogos", que não retratariam a repetição que o "consumidor-rei" deseja - mantendo, assim, um circuito viciado não só no reconhecimento de quem são os "gamers", mas também o que realmente contaria como “jogo".

\section{O Gamergate como rebote - Resistência identitária Nos Jogos Digitais}

Assim, embora historicamente calcada em uma série de circuitos culturais, compreendo que a "identidade gamer" masculina é contingente e performativa - ou seja, por mais que essa pareça estável (e tenha elementos potentes para essa estabilização - o que faz dela hegemônica), ela pode falhar a qualquer momento (BUTLER, 2003). São

\footnotetext{
8 "Comunidades de sedução" são grupos de homens que treinam "maneiras infalíveis" de seduzir mulheres. $\mathrm{Na}$ maioria das vezes, essas maneiras incluem crenças pseudocientíficas de acesso a "gatilhos psicológicos femininos" inconscientes, que fariam com que essas pudessem ser manipuladas para acesso sexual.
} 
essas falhas que possibilitam o acesso de outras populações e posições de sujeito a essa mídia - visto que, historicamente, a diversidade já é presente nessas culturas.

Assim sendo, reconheço as chamadas cenas indie queer de jogos digitais como um importante ponto de reconhecimento de diferença dentro da cultura de jogos digitais. Essas cenas são comunidades (on e off-line) que fazem jogos pessoais tendo como objetivo a constituição de uma cultura de jogo digital não mais centrada nessas experiências masculinas de guerra, dominação e na repetição incessante de mecânicas mas em possibilidades pessoais de liberdade artística, expressão, comunidade e desejo (FANTONE, 2014). Reconhecidas inicialmente em 2013 (KEOGH, 2013), centradas principalmente na cidade de Oakland, na Baía de São Francisco e na figura de game designers como Anna Anthropy, Mattie Bryce e Merrit Kopas; essa cena tinha como objetivo ser um espaço de troca de experiências (tanto técnicas quanto de vida) e produção cultural sobre vivências LGBTQ (na maioria das vezes centrado em pessoas trans) (KOPAS, 2015). Contudo, para mais do que a simples "representação", ou ainda a causa da "diversidade", esses movimentos compreendem que ali existe a possibilidade de expressar experiências diversas, organizar comunidades diferenciais e criar impactos artísticos e políticos. Anna Anthropy (2012) argumenta que a progressiva complexificação dos jogos (principalmente dos consoles, demonstrado pelas diferenças nos Joysticks desde a terceira geração), assim como o intenso investimento (monetário e de tempo) necessário para o desfrute da mídia mainstream acabam por afastar o público leigo, enquanto o mercado mobile ao mesmo tempo em que investe em acessibilidade, investe muito pouco em compreensões, narrativas ou mecânicas complexas ou inventivas. Assim, Anthropy e as demais pessoas do movimento têm como objetivo não apenas a "representação" de pessoas LGBTQ, mas criar, remanejar e pensar jogos que ofereçam experiências diferenciais, possibilidades de expressão outras e uma alternativa ao videogame mainstream, centrando na inventividade, criatividade e intimidade. Mais do que isso, esses movimentos acabam organizando-se para apontar o quanto essa diferença já está presente dentro das culturas, embora performativamente negada pela necessidade de manutenção da hegemonia "nerd masculina". Para tanto, essa cena serve-se e identifica-se como queer: um movimento sobre gênero, sexualidade, raça e outras 
estruturas de diferença e/ou populações oprimidas que renegam o multiculturalismo liberal e assimilacionista, tendo como objetivo minar as próprias estruturas que constroem as diferenças enquanto hierárquicas. Nesse sentido, essas cenas podem ser vistas como "herdeiras diretas" do Girls Games Movement, dos anos 1990 - grupos heterogêneos dispostos a repensar conteúdo, promoção, produção e alianças múltiplas. Tendo alguns membros afetados diretamente pela violência dos/as jogadores/as pertencentes ao movimento, os locais onde inicialmente essa cena se gestou se tornaram também locais de resistência intelectual e afetiva. A criação de termos / categorias como "SJW" acabaram por findar na composição de alianças antes impensadas, e aquilo que se iniciou como uma proteção conjunta contra um sistema opressor compôs então um coletivo produtivo antes improvável. A ideia de que essas pessoas estariam querendo "destruir os jogos digitais" foi incorporada, baseando importantes textos críticos sobre o assunto (ALEXANDER, 2014; PLUNKETT 2014) - além de influentes jams ${ }^{9}$ como a ironicamente nomeada Ruin Games 2014.

Não é mais possível explanar tais atos excludentes a uma "minoria barulhenta" ou ainda localizar esses impactos como efeito direto e vertical de uma "indústria" central - o Gamergate foi possível na cultura de jogo digital porque reflete uma série de organizações históricas de subjetivação próprias a esse meio. É, então, fundamental notar como mesmo boa parte das possibilidades de resistência - e sobrevivência - ao funcionamento opressivo dentro dos jogos digitais acaba por se compor de deslocamentos ou reapropriações de tendências que provém do próprio mainstream - ou ainda do uso de elementos históricos desse para reapresentar possibilidades outras de sujeito e de experiência, rompendo assim com a identidade gamer necessariamente ligada à sustentação hegemônica de homens, brancos, heterossexuais.

\footnotetext{
${ }^{9}$ Jams são encontros abertos, que tem como objetivo de criar jogos - das mecânicas até a programação (no caso de jogos digitais) ou confecção (no caso de jogos de tabuleiro) - durante o tempo da duração da Jam (na maioria das vezes um ou dois dias). As Jams costumam ser temáticas, sendo Queer Game Jams um importante fator na criação das cenas norte-americanas de jogos queer
} 
Referências

AHMED, Saeed, MARCO, Tony. Anita Sarkeesian forced to cancel Utah State speech after mass shooting threat. CNN, 2014. Disponível em http://edition.cnn.com/2014/10/15/tech/utah-anitasarkeesian-threat/. Acessado em novembro de 2017

ALEXANDER, Leigh. 'Gamers' Don't Have To Be Your Audience. 'Gamers' Are Over. Gamasutra, 2014.2 Disponível em http://www.gamasutra.com/view/news/224400/Gamers dont have to be your audience Gam ers are over.php. Acessado em junho de 2017

ALMOG, Ran; KAPLAN, Danny. The Nerd and His Discontent The Seduction Community and the Logic of the Game as a Geeky Solution to the Challenges of Young Masculinity. Men and Masculinities, 2015 p. $1-22$

ANTHROPY, Anna. Rise of the Video Game Zinisters: How Freaks, Normals, Amateurs, Artists, Dreamers, Dropoouts, Queer, Housewives, and People Like You Are Taking Back An Art Form. Nova York: Seven Stories Press, 2012.

AMARAL, Sílvia Cristina Franco; DE PAULA, Gustavo Nogueira. A nova forma de pensar o jogo, seus valores e suas possibilidades. Pensar a Prática, v. 10, n. 2, p. 155-168, 2007.

MIGUEL, Anna de; BOIX, Montserrat. Os Gêneros da Rede: os ciberfeminismos. In: NATAHNSON, Graciela. Internet em Código Feminino. Buenos Aires: La Crujía Ediciones, 2013.

BURGESS, Jean; MATAMOROS-FERNÁNDEZ, Ariadna. Mapping sociocultural controversies across digital media platforms: one week of\# gamergate on Twitter, YouTube, and Tumblr. Communication Research and Practice, v. 2, n. 1, 2016, p. 79-96.

BURNS, Matthew Seiji. The King and His Objects. Magical Wasteland, 2014. Disponível em https://www.magicalwasteland.com/notes/2014/8/22/the-king-and-his-objects. Acessado em junho de 2017.

BURRILL, Derek A. Die Tryin': Videogames, Masculinity, Culture. Nova York: Peter Lang Publishing. 2008.

BUTLER, Judith. Problemas de gênero: feminismo e subversão da identidade. Civilização Brasileira, Rio de Janeiro, 2003.

CASSEL, Justine, JENKINS, Henry. From Barbie to Mortal Kombat: Gender and Computer Games, MIT Press, Cambridge, 1998

CHESS, Shira; SHAW, Adrienne. A conspiracy of fishes, or, how we learned to stop worrying about\# Gamergate and embrace hegemonic masculinity. Journal of Broadcasting \& Electronic Media, v. 59, n. 1, p. 208-220, 2015.

CONDIS, Megan. No Homosexuals in Star Wars? BioWare, 'gamer' identity, and the politics of privilege in a convergence culture. Convergence, The International Journal of Research into

New Media Technologies. 2014. Pp. $1-15$

DONOVAN, Tristan. Replay: The history of video games. Yellow Ant, 2010.

FANTONE, Laura. Video Games and Queerness in California. Digital Creativity, v. 25, n. 4, 2014 , pp. $357-359$.

FRASCA, Gonzalo. Simulation versus narrative. Em: WOLF, Mark J.P., PERRON, Bernard. The video game theory reader, 2003. p. 221-235.

GERAÇÃO GAMER. Geração Gamer foi ameaçado por usuários do 55chan; pessoas também foram difamadas. Geração Gamer, 2015. Disponível em https://geracaogamer.com/2015/07/09/geracao-gamer-foi-ameacado-por-usuarios-do-55chanpessoas-foram-ameacadas/. Acessado em junho de 2017.

GOULART, Lucas Aguiar, HENNIGEN, Inês. Condições e Possibilidades de uma Tecnopolítica de Gênero/Sexualidade. Estudos Feministas, n. 22, v. 1, 2014, pp. 215 - 236. 
HARAWAY, Donna. Manifesto ciborgue: ciência, tecnologia e feminismo-socialista no final do século XX. In: SILVA, T. (Org.). Antropologia do ciborgue: as vertigens do pós-humano. Autêntica, Belo Horizonte, 2000.

HAYLES, N. Katherine. How We Became Posthuman. Chicago: University of Chicago Press, 1999.

IMHOF, Margarete; VOLLMEYER, Regina; BEIERLEIN, Constanze. Computer use and the gender gap: The issue of access, use, motivation, and performance. Computers in human behavior, v. 23, n. 6, p. 2823-2837, 2007.

IVORY, James .D. Still a Man's Game: Gender Representation in Online Reviews of Video Games. Mass Communication \& Society v. 9, n. 1, 2006 pp. 103-114.

JAGODA, Patrick . Videogame Criticism and Games in the Twenty-First Century. American Literary History, v. 29, n. 1, 2017, p. 205-218.

JAYANTH, Meg . 52\% of gamers are women - but the industry doesn't know it. The Guardian, 2014. Disponível em https://www.theguardian.com/commentisfree/2014/sep/18/52-percentpeople-playing-games-women-industry-doesnt-know. Acessado em junho de 2017

JUUL, Jesper. Half-real: Video Games between Real Rules and Fictional Worlds. Cambridge (Massachusetts) and London: OLDENBURG, 2005.

. A Casual Revolution: Reinventing Video Games and Their Players. Mit Press, 2009.

KAFAI, Yasmin B, HEETER, Carrie, DENNER, Jill, SUN, Jennifer Y. Beyond Barbie and Mortal Kombat: New Perspectives on Gender and Gaming. Cambridge (Massachusetts) and London: MIT Press, 2008.

KAFAI, Yasmin b., RICHARD, Gabriela T., and TYNES, Brendesha M. Diversifying Barbie and Mortal Kombat: Intersectional Perspectives and Inclusive Designs in Gaming. Lulu.com, 2016.

KENDALL, Lori. Hanging out in the virtual pub: Masculinities and relationships online. Univ of California Press, 2002.

"White and nerdy": Computers, race, and the nerd stereotype. The Journal of Popular Culture, v. 44, n. 3, p. 505-524, 2011.

KEOGH, Brendan. Just making things and being alive about it: The queer games scene. Polygon, 2013. Disponível em https://www.polygon.com/features/2013/5/24/4341042/the-queer-gamesscene. Acessado em junho de 2017.

KOPAS, Merritt. Video Games for Humans: Twine Authors in Conversation. San Francisco: Instar Books. 2015.

LEE, Anthony. Veerender Jubal Photoshopped To Be Paris Atacker. Techraptor, 2015. Disponível em https://techraptor.net/content/veerender-jubal-photoshoped-paris-attacker. Acessado em junho de 2017.

MASSANARI, Adrienne. \# Gamergate and The Fappening: How Reddit's algorithm, governance, and culture support toxic technocultures. New Media \& Society, 2015, p. $1-18$.

MENDES, Claudio. Lúcio. Jogos Eletrônicos: Diversão, Poder e Subjetivação. Campinas: Papirus, 2006.

MORTENSEN, Torill Elvira. Anger, fear, and games: The long event of\# Gamergate. Games and Culture, 2016.

NAGLE, Angela. Kill All Normies: Online Culture Wars from 4chan and Tumblr to Trump and the Alt-right. Washington, D.C.: Zero Books, 2017.

PARTIDO PIRATA. Machismo na rede: ameaça de um gamergate brasileiro. Partido Pirata, 2015. Disponível em https://partidopirata.org/ameaca-de-gamergate-brasileiro-uma-reprisepara-nao-ver/. Acessado em junho de 2017.

PERREAULT, Gregory P.; VOS, Tim P. The Gamergate controversy and journalistic paradigm maintenance. Journalism, 2016, p 1 - 17 
PESQUISA GAME BRASIL, Pesquisa Game Brasil, 2016. Disponível em https://www.pesquisagamebrasil.com.br/. Acessado em junho de 2107.

PLANT, Sadie. A Mulher Digital. Editora Rosa dos Tempos, Rio de Janeiro, RJ, 1997.

PLUNKETT, Luke. We Might Be Witnessing a 'Death Of An Identity'. Kotaku, 2014. Disponível em http://kotaku.com/we-might-be-witnessing-the-death-of-an-identity-1628203079. Acessado junho de 2017

PORTAL IMPRENSA. Após escrever texto contra machismo, blogueira do Brasil Post é vítima de ameaças . Portal Imprensa, 2015. Disponível em http://www.portalimprensa.com.br/noticias/brasil/71015/apos+escrever+texto+contra + machism o+blogueira+do+brasil+post+e+vitima+de+ameacas. Acessado em junho de 2017.

ROLNIK, Suely. Toxicômanos de Identidade: subjetividade em tempo de globalização. In: LINS, D. (Org.). Cultura e subjetividade: saberes nômades. Campinas. Papirus, 1997

RYERSON, Liz. Embracing The New Flesh. Ellaguro, 2014. Disponível em http://ellaguro.blogspot.com.br/2014/10/embracing-new-flesh.html

SALTER, Michael. From geek masculinity to Gamergate: the technological rationality of online abuse. Crime, Media, Culture, 2017, p. 1 - 18.

SHAW, Adrienne. Gaming at The Edge: Sexuality and Gender at the Margins of Videogame Culture. Minneapolis: University of Minnesota Press, 2014

Do you identify as a gamer? Gender, race, sexuality, and gamer identity. new media $\&$ society, v. 14, n. 1, p. 28-44, 2012.

SQUIRE, Kurt. From Content to Context: Videogames as Designed Experience. Educational Researcher, Vol. 35, No. 8, 2006, pp. 19 - 29.

TOTLO, Stephen. Another Woman In Gaming Flees Home Following Death Threats. Kotaku, 2014. Disponível em http://kotaku.com/another-woman-in-gaming-flees-home-following-deaththre-1645280338. Acessado em junho de 2017.

WARNER, Michael. Publics and counterpublics. Public culture, v. 14, n. 1, p. 49-90, 2002.

ZIMMERMAN, Eric, CHAPLIN, Heather. Manifesto: The 21st Century Will Be Defined By Games. Kotaku, 2013. Disponível em http://kotaku.com/manifesto-the-21st-century-will-bedefined-by-games-1275355204 Acessado em junho de 2017. 